\title{
REVISÃO DOS INSTRUMENTOS DE DIAGNÓSTICO MÉDICO-FORENSE NO ALCOOLISMO
}

\author{
REVIEW OF MEDICAL FORENSIC DIAGNOSTIC INSTRUMENTS ON ALCOHOLISM
}

\author{
Roberto Augusto de Carvalho Campos* \\ Rosmari Aparecida Elias Camargo*
}

Resumo:

A elevada prevalência mundial do alcoolismo, associada às graves conseqüências orgânicas do consumo etílico, fazem dessa doença um grande problema social com significativas repercussões no Direito.

Nas mais diversas áreas do Direito, a atividade jurisdicional depende do preciso dimensionamento da extensão e magnitude das lesões nos órgãos e sistemas, cabendo à Medicina Forense sistematizar os critérios e instrumentos diagnósticos da doença. Os exames de investigação mais adequados para esse estudo são discutidos no presente trabalho, com destaque para suas indicações e limitações.

Palavras-chave: Alcoolismo. Testes psicológicos. Tomografia computadorizada. Ressonância magnética. Doença mental.

\begin{abstract}
:
The high global prevalence of alcoholism associated with severe organic consequences of alcohol consumption, make this disease a major social problem with significant impact on Law. In many areas of law, judicial activity depends on the accurate sizing of the extent and magnitude of damage to the organs and systems, falling to Forensic Medicine systematize the criteria and diagnostic instruments of this disease. The exams of investigation most appropriate for this study are discussed in this work, with emphasis on their indications and limitations.
\end{abstract}

Keywords: Alcoholism. Psychological tests. Computed tomography. Magnetic ressonance image. Mental disease.

\section{Introdução}

O alcoolismo tem acompanhado a história da humanidade com marcantes danos ao homem, à sua saúde e à sociedade. Fatores culturais, tolerância social, ampla disponibilidade, baixo custo, sensação de prazer e de alívio frente à angústia induzem aqueles que têm predisposição a um consumo sistemático.

\footnotetext{
Professor Doutor do Departamento de Direito Penal, Medicina Forense e Criminologia da Faculdade de Direito da Universidade de São Paulo. Mestre e Doutor em Medicina pela Universidade Federal de São Paulo - Escola Paulista de Medicina. http://www.camargoecampos.com.br.

** Mestre em Direito Penal pela Faculdade de Direito da Universidade de São Paulo.
} 
O álcool é produto da fermentação da glicose encontrada nas frutas, cereais e tubérculos, sob a influência de fermentos que são os saccharomyces. (SZNICK, 1987)

Os danos sociais causados pelo alcoolismo são significativos. Nos países desenvolvidos, a incidência de indivíduo alcoolizado ocorre em 50\% dos acidentes automobilísticos fatais, 50\% dos homicídios, 30\% dos estupros, 25\% dos suicídios e mais de $60 \%$ de casos de violência contra crianças, sendo, ainda, causa de alta prevalência nas hemorragias digestivas, nos traumas cranioencefálicos por quedas e acidentes domiciliares. (WALKER, 1983; linnoila e cols., 1986)

A prevalência de alcoolismo chega a ser de $15 \%$ entre os homens e de $5 \%$ entre as mulheres. (Andrade \& Hirata, 1985). Nos Estados Unidos da América, o alcoolismo ocorre em 5 a 15\% dos homens, e em 3 a 5\% das mulheres. (SCHUCKIT, 1982)

A Secretaria Nacional Antidrogas, da Presidência da República do Brasil, em 2002 publicou a Política Nacional Antidrogas, ${ }^{1}$ apontando que o uso indevido de drogas constitui, na atualidade, séria e persistente ameaça à humanidade e à estabilidade das estruturas e valores políticos, econômicos, sociais e culturais de todos os Estados e sociedades.

Tais repercussões sociais da doença alcoolismo determinam especial atenção da Medicina Forense no sentido de buscar uma adequada utilização dos modernos instrumentos diagnósticos disponíveis. E ainda, as elevadas prevalências do alcoolismo, aliadas aos graves danos causados no organismo humano, ensejam o entendimento do caráter endêmico da doença. O prejuízo social direto e o interesse da Medicina referente ao alcoolismo é traduzido pela ampla literatura sobre esse tema. Indiretamente, a sociedade padece a necessidade de contemplação jurisdicional de tão grave flagelo. É função da pesquisa médica subsidiar o Direito, no sentido de determinar precisamente a magnitude das lesões provocadas pelo álcool e, em última análise, possibilitar o diagnóstico da inimputabilidade jurídica.

O presente trabalho tem como objetivo identificar e analisar os critérios, a adequação e a indicação dos métodos diagnósticos periciais tradicionais bem como aqueles de moderna tecnologia.

\section{Aspectos históricos a respeito da produção e consumo de bebida alcoólica}

Os avanços das técnicas de agricultura no mundo possibilitaram o aparecimento de grandes áreas produtoras, como na Índia e no Egito. A cultura de arroz e de cevada, as boas condições ambientais como: abundância de água de boa qualidade; região de clima quente e açúcar das frutas sempre disponíveis viabilizaram a produção da

D.O.U. $165-27.08 .2002$ 
cerveja, tornando-a a primeira bebida alcoólica produzida em larga escala, por volta de 3400 a.C. (JELLINEK, 1946) Meio século após, surgem no Egito indícios da produção de vinho e, na Grécia, ela ocorre somente a partir de 1000 a.C. (GUEBALY, 1981)

As primeiras referências médicas sobre o alcoolismo surgiram aproximadamente no ano 4000 a.C., também na Grécia com Hipócrates, que relacionou o quadro clínico de tremor e convulsão à ingestão crônica de bebidas alcoólicas. (GUEBALY, 1981)

Desde os primórdios da produção em larga escala de bebidas alcoólicas, surgiu a preocupação do controle, através de instrumentos legais. Hamurabi, 1793 a 1759 a.C., sexto rei da primeira dinastia da Babilônia, criou o chamado Código de Hamurabi com 282 artigos de caráter restritivo ao consumo de álcool e sua comercialização, regulando, inclusive, o funcionamento das tabernas persas. (KRAMER, 1983)

Embora os romanos tivessem conhecimento do cultivo da videira antes dos gregos, o consumo do vinho em maior escala ocorreu somente a partir de 200 anos a.C. Naquela época, o vinho era caro e escasso, bebia-se muito pouco e era proibido às mulheres. Após essa data se inicia a expansão do cultivo de uvas, com conseqüente aumento de produção do vinho. (JELLINEK, 1976)

A técnica de destilação foi criada no século XI, determinando mudança dos hábitos em vários países da Europa e, com o tempo, sua produção intensificou-se, sem a exclusão das bebidas fermentadas, levando a sociedade a um elevado consumo, o que gerou restrições progressivas por parte da Igreja e do Direito. (PACHECO \& SILVA, 1951)

Na América do Norte, a colonização encontrou produção de bebidas fermentadas pelos nativos. Os colonizadores introduziram os destilados, impondo aos indígenas, um consumo que se acentuou em pouco tempo. No Brasil, os portugueses encontraram bebidas fermentadas produzidas com mandioca e frutas como o caju. Com a cana-de-açúcar, produzida em larga escala no Nordeste, Rio de Janeiro e São Paulo, a aguardente de cana foi amplamente difundida entre a população incluindo indígenas e escravos, a quem os próprios senhores ofereciam em ocasiões festivas, provando que, no Brasil, a preferência pelos destilados surgiu nos tempos coloniais. (FORTES, 1975)

Os problemas sociais em larga escala relativos ao alcoolismo surgiram somente no século XX, com a promulgação da "lei seca" nos Estados Unidos da América, em 1919, que proibia o consumo de álcool. Nos primeiros anos da vigência dessa lei, o consumo do álcool e suas conseqüências diminuíram, mas posteriormente, o crime e a corrupção reativaram os mesmos problemas, que cronificados, motivaram em 1935 a fundação, por ex-alcoólicos, da Sociedade Alcoólicos Anônimos, com o objetivo de reabilitar doentes viciados. (FORTES, 1975) 
As repercussões graves de saúde devidas ao alcoolismo têm resultado, em relação à Medicina, em extensas pesquisas a respeito desses efeitos no organismo humano, identificados em vários órgãos e sistemas assim como a interação da droga com células e tecidos e da relação causal com inúmeras afecções. (CORBETT, 1982; DIAMOND \& GORDON, 1997)

\section{Conceito e diagnóstico de alcoolismo}

O termo alcoolismo foi criado em 1849, por um médico sueco, chamado Magnus Huss, em um ensaio denominado em latim Alcoholismus chronicus, com a subfrase sueca "eller chronisk alkoholssjukdom", que significa "ou adição ao álcool". Embora com algumas falhas, o estudo feito por Huss trazia importantes informações acerca dos sintomas e lesões ocasionadas pela intoxicação crônica pelo álcool (ELKIS, 1985; KELLER, 1983)

Na metade do século XIX, utilizou-se pela primeira vez a expressão alcoolismo crônico. (FORTES \& GATTO, 1975) Em 1972, o "Criteria Commitee" do National Council on Alcoholism dos Estados Unidos da América realizou seminário para estabelecer critérios para o diagnóstico do alcoolismo. A conceituação efetiva ocorreu em 1976, com o "Committee on Definitions", pertencente ao "National Council on Alcoholism", em cooperação com o “American Medical Society on Alcoholism”, oportunidade em que o alcoolismo foi definido com ênfase na natureza progressiva, nas seqüelas físicas e nos fenômenos de tolerância e dependência.

Alcoolismo é uma doença crônica, progressiva e potencialmente fatal, caracterizada por tolerância e dependência física e/ou por manifestações orgânicas patológicas como conseqüência direta ou indireta de sua ingestão. (NATIONAL Council on Alcoholism e American Medical Society on Alcoholism, 1976)

Em 1992, o "National Council on Alcoholism and Drug Dependence" e a "American Society of Alcoholism and Addiction Medicine" conceituaram o alcoolismo como "doença crônica primária, cujo desenvolvimento e manifestações são influenciados por fatores genéticos, psicossomáticos e ambientais. Geralmente é uma doença progressiva e letal, caracterizada por comprometimento contínuo ou periódico do controle do hábito de beber, preocupação com o álcool, uso do álcool, apesar das conseqüências adversas e distorções do pensamento, principalmente negação". Os membros do grupo de estudo destacaram que o termo "primário" diz respeito a uma entidade isolada de outros estados patológicos. O caráter progressivo enfatiza o processo evolutivo da doença, bem como a persistência dos distúrbios físicos, emocionais e sociais, que são geralmente cumulativos e progridem com a persistência do uso. É progressivo e fatal, pois a doença perdura no tempo e as alterações físicas, emocionais e sociais são freqüentemente cumulativas. 
Existem conseqüências adversas que estão relacionadas à morbidade do álcool em relação a manifestações físicas, psicológicas, ocupacionais, legais, econômicas, espirituais ou pessoais. (MORSE e cols., 1992)

\section{Critério diagnóstico}

A American Psychiatric Association (1987) enfatizou que, para diagnosticar o alcoolismo, são necessários três dos sintomas relacionados:

- o álcool é consumido por período maior ou em quantidade maior do que o indivíduo pretende;

- desejo persistente de ingerir bebidas alcoólicas;

- gasta-se muito tempo para buscar o consumo do álcool ou para recuperar-se de seus efeitos;

- ocorre intoxicação ou sintomas de abstinência com freqüência;

- devido ao álcool, o indivíduo abre mão de atividades sociais, profissionais ou recreativas;

- ocorrem problemas sociais, psicológicos e físicos;

- tolerância;

- abstinência. (ABEL, 1998)

No Diagnostic and Statistical Manual Disorders (DSM-IV) de 1994, foram elencados sinais encontrados nos indivíduos com ingestão recente de álcool: alteração da fala, incoordenação, instabilidade da marcha, nistagmo, déficit na atenção e memória, torpor e coma. Em relação à abstinência alcoólica, o DSM-IV estabelece como critério diagnóstico, além da cessação ou redução no consumo, a presença de dois ou mais sinais dos seguintes: hiperatividade autonômica; alucinação ou ilusão visual, auditiva ou tátil; agravamento do tremor das mãos; insônia; náusea ou vômito; agitação psicomotora; ansiedade; convulsões. A maioria dos indivíduos com consumo elevado de álcool apresenta sinais e sintomas da abstinência, sendo que somente $5 \%$ dos alcoólicos nunca experimentaram tais efeitos.

A Classificação Internacional de Doenças e Problemas Relacionados à Saúde (CID-10), de 1997, englobou o alcoolismo nos transtornos mentais e comportamentais, devidos ao uso de substância psicoativa. Este agrupamento compreende numerosos transtornos que diferem entre si, pela gravidade variável e por sintomatologia diversa causada por substância psicoativa. Nessa classificação, determinam-se modalidades de consumo que podem ser relacionadas à:

- intoxicação aguda;

- síndrome (estado) de abstinência;

- transtorno psicótico;

- transtorno psicótico residual ou de instalação tardia. 


\section{Exames subsidiários no alcoolismo}

\subsection{Eletrofisiologia e eletroencefalograma}

O eletroencefalogram - EEG registra a atividade elétrica das camadas da cortiça cerebral que se encontram adjacentes ao crânio. É universalmente aceito que a atividade do EEG representa as alterações graduadas de potencial nos dendritos aglomerados dos neurônios corticais. A despeito de sua origem cortical superficial, a forma do EEG normal depende dos estímulos que chegam ao córtex, provenientes de estruturas cerebrais profundas. (BRAZIER, 1964)

A característica proeminente do EEG no indivíduo adulto normal desperto é o ritmo alfa, com freqüência de 8 a 13 ciclos por segundo, que é mais proeminente nas porções posteriores. (BRAZIER, 1964)

A atividade alfa tem uma amplitude de 1 a 100 microvolts (média de 25 a 50) e, embora essa amplitude possa diferir substancialmente entre os hemisférios, a freqüência alfa é normalmente idêntica em ambos. (GLASS, 1964)

A freqüência do ritmo alfa é geralmente bem constante para um determinado indivíduo. Contudo, a freqüência alfa diminui com alterações metabólicas, como ocorre no alcoolismo. (GLASS, 1964)

Outro ritmo encontrado no EEG é a atividade beta, com freqüência maior que 13 ciclos por segundo. Ela aparece em todos os registros de adultos normais, sendo mais proeminente nas áreas frontal e central. Normalmente, é de baixa amplitude, com menos de 20 microvolts. O ritmo beta predomina em alguns indivíduos normais e freqüentemente substitui a atividade alfa nos pacientes tensos e ansiosos. (ROMANO \& ENGEL, 1944)

As ondas teta têm intensidade menor do que 20 microvolts em amplitude. São mais proeminentes nas áreas fronto-temporais e ocupam até 5\% de alguns EEGs normais. (SILVERMAN, 1962)

As ondas delta são mais lentas do que 4 ciclos por segundo e não ocorrem no EEG normal do adulto. (SILVERMAN, 1963)

Já que a atividade eletroencefalográfica normal depende da integridade do córtex e estruturas profundas, deduz-se que o exame pode estar alterado por disfunção dos neurônios corticais; por distúrbios de estruturas do diencéfalo ou tronco cerebral, influenciando o EEG ou por interrupção de conexões entre o córtex e as estruturas encefálicas mais profundas. (BRAZIER, 1964)

Os centros neurais que influem sobre a consciência tendem a ter efeito importante no EEG e, em sua maioria, condições que alteram a consciência, alteram também o resultado do exame. (SILVERMAN, 1963) 
A doença cerebral de etiologia metabólica produz traçado de EEG com lentificação simétrica, difusa, em proporção direta à gravidade da encefalopatia. Mesmo o mais leve delírio é usualmente acompanhado por redução da freqüência alfa. (GLASS, 1964)

Quando o paciente se torna letárgico, verifica-se a presença de salvas paroxicísticas de ondas sincrônicas, bilaterais, de alta voltagem, com freqüência de 2 a 4 ciclos por segundo, que se espraiam lateral e posteriormente, conforme a depressão cerebral se aprofunde. Estas ondas paroxicísticas podem adotar várias formas como, por exemplo, a espícula onda. Ocorre com mais freqüência na encefalopatia hepática, freqüente no alcoolismo e pensou-se em primeira instância que era característica dessa desordem. (SILVERMAN, 1962)

No coma profundo, o traçado do EEG consiste de atividade lenta, com freqüência de 1 a 3 ciclos por segundo, difuso e de alta voltagem (SILVERMAN, 1963)

Embora o EEG seja sempre lento nos comas metabólicos, a recuperação eletroencefalográfica pode ser retardada, comparada com a recuperação clínica, podendo persistir os registros muito lentos, semelhantes aos do coma, durante dias ou semanas depois que o paciente desperta. (SILVERMAN, 1963)

O estudo funcional do cérebro foi realizado por autores como ZILM e cols. (1980) que estudaram os distúrbios eletroencefalográficos presentes nos pacientes com diagnóstico de Síndrome Psicorgânica Alcoólica. Os autores observaram que a maioria dos pacientes não apresentou nenhuma alteração no exame, identificando em alguns, irregularidades de atividade alfa, beta e teta, podendo ocorrer o aparecimento de espícula ondas. Nos casos em que a doença evoluiu para deterioração acentuada da função mental, o eletroencefalograma (EEG) veio apresentar-se com lentificação generalizada com a presença de ondas delta e teta. Os autores lembram, ainda, que as anomalias encontradas ao EEG não dependem do tempo do uso de álcool, da quantidade ingerida, bem como do tipo de bebida utilizada.

Ishibashi et al. (1987), ao avaliarem alcoolistas em abstinência, através da tomografia computadorizada e EEG, concluíram que com a abstinência há diminuição da atrofia cerebral e do traçado eletroencefalográfico para alguns pacientes. Os autores estabeleceram correlação entre os dois eventos, considerando que as alterações do EEG, observadas nos indivíduos alcoolistas, está associada com a atrofia cerebral diagnosticada pela TC.

Krauss \& Niedermeyer, em 1991, realizaram EEGs em pacientes alcoolistas, com episódios relatados de convulsão. Desses pacientes, 90\% apresentaram crises convulsivas tônico-clônicas generalizadas e metade dos eventos convulsivos ocorreram em período de início de abstinência, qual seja, de 8 horas a 7 dias de abstinência e, naqueles pacientes que apresentaram crises convulsivas parciais, identificou-se algum 
tipo de lesão estrutural à tomografia computadorizada ou referência de trauma craniano. Constataram ainda os autores que a maioria dos pacientes estudados apresentou traçado eletroencefalográfico com ondas de baixa voltagem.

Em 1980, Zilm e cols., ao realizarem eletroencefalograma em indivíduos alcoolistas, não observaram alterações significativas nos traçados obtidos. Somente naqueles que apresentavam grande deterioração mental é que foram observadas alterações compatíveis com sofrimento cerebral.

\subsection{Potenciais evocados}

Três tipos de potenciais evocados são utilizados na rotina clínica:

a) potencial evocado auditivo;

b) potencial evocado somato-sensitivo;

c) potencial evocado visual.

Essa modalidade de exame visa, após estímulo auditivo, sensitivo (táctil) ou visual, a captação dos potenciais de ação provocados ao longo das respectivas vias até o córtex cerebral. Assim, qualquer tipo de interrupção destas vias ou interferência nas mesmas irá provocar alteração destes potenciais, não só em relação à sua amplitude, mas também ao tempo de latência.

Dom Zal et al., em 1989, realizaram exames de potenciais evocados auditivos, visuais e somatosensitivos em alcoolistas. Obtiveram como achados, alterações significativas nos exames auditivos que reputaram estar relacionadas com disfunções funcionais do cerebelo. Por outro lado, em relação aos potenciais evocados visuais e somatosensitivos, as alterações nos exames foram menos evidentes.

As respostas auditivas de tronco encefálico foram utilizadas experimentalmente, para avaliar alterações eletrofisiológicas relacionadas com a ingestão de álcool. CHU e cols. (1978) observaram o aumento da latência das ondas III, IV, V e VII. Achados semelhantes encontraram em humanos, concluindo que o álcool afeta as estruturas centrais do sistema nervoso central e não o órgão receptor perifericamente. (SQUIRES e cols., 1978; SQUIRES e cols., 1978).

\subsection{Alcoolismo e respostas auditivas}

Os mecanismos de ação do álcool no sistema nervoso central não são ainda completamente conhecidos. (SALAMY, 1973). Como descrito anteriormente, as alterações crônicas do álcool sobre o tecido nervoso levam a alterações comportamentais que são relativas à atividade neuronal. Estudos biofísicos revelam que doses elevadas de etanol inibem a atividade neuronal pela hiperpolarização da membrana celular pelo aumento 
da condutância do potássio, possivelmente por elevar os íons de cálcio livre intracelular (LOVINGEN, 1993).

As respostas auditivas encefálicas são modalidades de exames disponíveis para avaliação integrada da eletrofisiologia e cognição. Tal técnica consiste em captar a resposta elétrica cerebral, após estímulos sensórios repetidos de várias naturezas. Este volume de condução pode ser captado devido à orientação geométrica dos neurônios, que produzem um campo elétrico que pode ser detectado remotamente (HALL III, 1990). Os componentes mais precoces, que têm duração de até 100 milisegundos, refletem as características próprias do estímulo, enquanto os componentes mais tardios são influenciados por fatores psicológicos. (PORJEZZ \& BEGLEITER, 1989; BEGLEITER e cols., 1981).

Apesar de que as ondas (discriminadas abaixo por algarismos romanos) possam ser reflexos de atividade neuronal de vários sítios, seus principais geradores são: I - nervo coclear; II - núcleo coclear; III - complexo olivar superior; IV - lemnisco lateral; V - colículo inferior; VI - corpo geniculado medial; VII - radiações talâmicas. Essas topografias são identificadas no exame, como deflexões positivas que são analisadas na amplitude e latência das ondas. As ondas I e II são componentes do ABR que não tem múltiplos sítios geradores, ou seja, o pico de onda é específico para a estrutura anatômica indicada. (BUCHWALD \& HUANG, 1975; STOCKARD \& ROSSITER, 1977)

Os potenciais de média latência ocorrem entre 12 e 50 milisegundos e surgem logo após os potenciais de tronco encefálico. Neste exame, podem-se identificar potenciais no corpo geniculado medial; cápsula interna; núcleo lentiforme; cápsula externa; ínsula e córtex auditivo no giro de Heschl. Os potenciais de média latência não possuem sítios específicos de geração, refletem ativação global cortical e subcortical. A deflexão Pa é a mais constante e também visível, ocorrendo o seu pico em 25 milisegundos, com amplitude de 1,0 microvolts. Seu sítio de origem é o tálamo e córtex auditivo, tendo sua análise maior valor diagnóstico. (GANANÇA, 1998) Os potenciais de média latência sofrem influência de fatores como o estado de vigília, sedação, idade, sexo e agentes depressores do sistema nervoso central - SNC. Por esses motivos, os exames eletrofisiológicos devem ser realizados em indivíduos descansados, afastando-se uso de drogas depressoras do SNC pelo menos 24 horas antes da realização do teste.

A resposta auditiva de tronco encefálico é indicada para diagnósticos e evolução das doenças da fossa posterior, hipóxia, anomalias metabólicas, trauma, hemorragia subaracnóidea, infartos de tronco, doenças desmielinizantes, maturação encefálica das crianças, avaliação objetiva de perda auditiva, avaliação do coma e monitorização de cirurgias da fossa posterior. Destacam-se três indicações clínicas para a realização das respostas auditivas de média latência: diagnóstico topográfico de lesões de qualquer etiologia na região subcortical e cortical; monitorização da evolução das doenças 
que afetam essas áreas; acompanhamento da eficácia de seus respectivos tratamentos; determinação dos limiares auditivos. (GANANÇA, 1998)

Em 1973, Salamy \& Williams, realizando a análise dos potenciais evocados somato-sensitivo em humanos, não encontraram qualquer alteração dos potenciais dependentes do consumo de álcool. Experimentalmente, Bierley e cols., em 1980, analisando ratos submetidos à ingestão crônica de álcool, observaram a diminuição da amplitude das ondas e da latência dos potenciais evocados visuais.

Hyvarinen e cols., em 1978, com trabalho experimental e Begleiter e cols., em 1981, afirmaram ocorrerem, pelo efeito do álcool, alterações neuronais funcionais captadas pelos potenciais tardios, isto devido à complexidade das conexões sinápticas existentes. As evidências mostram que os sítios polissinápticos cerebrais, como áreas subcorticais e formação reticular, são extremamente sensíveis ao álcool.

De acordo com Salamy e cols. (1973), as alterações elétricas podem ocorrer em tempos variáveis após a ingestão de álcool e acrescentaram que o consumo de doses pequenas que levariam a uma concentração sérica de 30 a 65mg/100ml, não produzem alterações significativas nos potenciais evocados auditivos. Doses que atinjam concentração de 90 a $110 \mathrm{mg} / 100 \mathrm{ml}$ interferem significativamente na amplitude das ondas, apresentando variação diretamente proporcional ao aumento da dose com a alteração da amplitude da onda. Segundo estes autores, mesmo se considerando variações individuais no metabolismo do etanol, pode-se inferir, pela baixa amplitude das ondas, o quanto foi ingerido.

Begleiter \& Porjesz, em 1977, experimentalmente, observaram que os animais, após longo período de consumo de álcool, quando entram em abstinência, vão apresentar aumento da voltagem dos potenciais de ação, o que caracteriza, segundo os autores, estado de hiperexcitabilidade elétrica cerebral; cujo efeito rebote, segundo Peeke e cols. (1979), já ocorre com uma semana de abstinência, podendo perdurar por três a quatro semanas e não deve ser confundido com os efeitos de drogas, que também elevam a amplitude dos potenciais. Por tais motivos, eliminamos do nosso estudo os indivíduos em abstinência superior a dez dias, bem como os que fazem uso de qualquer tipo de medicamento. Em tese, esta fase de hiperexcitabilidade elétrica pode, conseqüentemente, mitigar disfunções elétricas do sistema nervoso central, pois eventuais diminuições de amplitude dos potenciais de ação estariam compensadas pela transitória hiperexcitabilidade reativa. (SQUIRES e cols., 1978).

Em 1981, Begleiter e cols. discutiram novamente estes achados, afirmando que nos potenciais evocados de tronco cerebral ocorre aumento de latência do interpico II-V. Os autores pesquisados destacam duas modalidades de alteração elétrica que são o aumento de latência e baixa voltagem dos potenciais de ação. Descreveram, ainda, o aumento da latência das ondas II, III, IV e V, sugerindo que o tempo de transmissão do 
estímulo no tronco seria mais longo nos alcoólicos do que no grupo controle. Os autores afirmaram que tais evidências mostram que o abuso do álcool resulta em déficits de condução no tronco cerebral, sugerindo possível desmielinização dos tratos auditivos.

Este processo de desmielinização é comprovado por estudos neuroquímicos que evidenciaram alteração da composição lipídica da substância branca cerebral, com aumento do conteúdo hídrico. Estes achados são evidentes, além da condição de desmielinização, também na degeneração walleriana. (HARPER e cols., 1988)

Segundo Realmuto e cols. (1993), podemos encontrar como efeito agudo do consumo de álcool, o aumento da latência da onda. Cessado o efeito agudo, encontram-se, independentemente das alterações elétricas agudas, outras consideradas crônicas que são as relacionadas com a diminuição da amplitude dos potenciais. Jääskelaïnen e cols., em 1995, relataram estudos que encontraram latência aumentada com a administração aguda de etanol, nas respostas auditivas de tronco encefálico.

Na síndrome de abstinência ocorre hiperexcitação cerebral, causando resposta auditiva eletrofisiológica acelerada. (BEGLEITER \& PORJESZ, 1977; PEKRONEN e cols., 1998) Apesar disso, as informações auditivas são processadas da mesma maneira como nos indivíduos não expostos ao álcool, ou seja, a informação é processada no córtex auditivo contralateral mais cedo que o ipsilateral, pois a maioria das fibras do sistema auditivo cruza a linha média e ascendem ao córtex auditivo contralateral, sendo que uma proporção relativamente pequena de axônios alcança o córtex auditivo ipsilateral.

$\mathrm{O}$ estudo de potenciais mais tardios justifica-se por trabalhos que apontam para o achado de atrofia cortical e subcortical demonstráveis por exames neuroradiológicos e necroscópicos (MILNER, 1963; LUZINSE e cols., 1980; LEE e cols., 1982). Ron, em 1977, relatou que o lobo frontal é mais afetado no cérebro, salientando que os maiores danos estão localizados em áreas corticais e subcorticais.

Com isso, ao analisarmos as respostas auditivas de média latência objetivamos maior acuidade do exame, justamente por se poderem abordar áreas onde as alterações radiológicas e necroscópicas são mais evidentes.

Tais alterações manifestam-se predominantemente com a abolição de potenciais, ou seja, ocorreu a ausência de potenciais detectáveis pelo equipamento utilizado.

Os resultados alterados encontrados nas respostas auditivas de média latência nos indivíduos alcoólatras justificam-se devido às possíveis lesões da microcirculação terminal dos sítios geradoras dos potenciais. As características particulares de irrigação da região cortical e subcortical tornam essas áreas suscetíveis à redução de fluxo, proporcionando distúrbios na neurotransmissão, cujos efeitos foram identificados pelas respostas auditivas de média latência. Não se encontram alterações semelhantes nas 
respostas auditivas de tronco encefálico provavelmente porque a circulação do tronco encefálico tem abundância de vasos sanguíneos colaterais para cada área irrigada.

Outros potenciais tardios, como o P300, devido a sofrer grande influência de variáveis importantes como a idade, estado emocional, cansaço físico, atenção e vigília, que poderiam alterar com significância os nossos resultados. Quanto à idade, Realmuto e cols. (1993) lembram sua influência na latência das ondas, que vai apresentar-se prolongada.

Pfefferbaum e cols. (1979) identificaram nas respostas auditivas de tronco encefálico, diminuição da amplitude das ondas com latência de 100 a 250 mseg.

Begleiter e cols. (1981) estudaram em alcoólicos crônicos em abstinência as respostas auditivas de tronco encefálico e encontraram as latências das ondas II, III, IV e $\mathrm{V}$ significativamente aumentadas, quando comparadas com o grupo controle, denotando tempo de transmissão do estímulo no tronco cerebral prolongado nestes indivíduos. Os autores sugerem neste trabalho que o abuso crônico do consumo de álcool provoca processo de desmielinização do trato auditivo.

Church \& Williams (1982), ao pesquisarem os efeitos agudos do etanol nas respostas auditivas de tronco encefálico, encontraram resultados não uniformes, apesar dos achados predominantes de aumento do tempo de latência das ondas sem levar a alterações da amplitude. Essas alterações, identificadas na onda II, devem refletir a ação do etanol nos núcleos cocleares.

Em 1993 e posteriormente em 1996, Nichols \& Martin analisaram as respostas eletrofisiológicas pelo P300, encontrando alterações de latência e amplitude de potenciais.

A análise comparativa dos potenciais evocados tardios e a tomografia computadorizada de indivíduos alcoolistas, evidenciou existir concomitância de traçados alterados com anormalidades tomográficas do córtex frontal. (OGURA \& MIYAZATO, 1991)

\subsection{Diagnóstico por imagem}

O diagnóstico das complicações encefálicas nos alcoólicos pela imagem radiológica teve acentuado avanço com o advento da tomografia computadorizada (TC) e ressonância magnética (RM). No entanto, como observamos na literatura, o estudo por imagem das alterações morfológicas encontradas nesses exames, revelam sinais inespecíficos que podem não refletir o real comprometimento do Sistema Nervoso Central (SNC). (LEE e cols., 1979; LUSINS e cols., 1980; LEE e cols., 1982; WEST e cols., 1984)

Nessa modalidade de diagnóstico, Barini \& Silva (1959) realizaram trabalho inédito ao estudarem o pneumoencefalograma em alcoólicos. Brewer \& Perret (1981), 
também com o uso do pneumoencefalograma, procuraram correlacionar as alterações atróficas cerebrais encontradas no exame com possível deterioração mental causada pelo álcool. Esta associação, que se mostrou positiva, foi também quantificada por estudo psicométrico e eletroencefalográfico.

\subsubsection{Tomografia computadorizada}

O início do uso clínico da TC ocorreu no ano de 1972, quando Hounsfield (1973), pesquisador inglês da indústria EMI Ltda., desenvolveu o primeiro tomógrafo que foi instalado em Wimbledon, no hospital Atkinson Morley.

A aquisição de imagem da tomografia computadorizada é realizada por um ou vários tubos de irradiação de raios-x, que executam movimentos de rotação ao redor do segmento corpóreo a ser estudado. Os raios-X assim emitidos sensibilizam cristais detectores, produzindo sinais que são digitalizados e captados por um computador que recupera, em matriz, a imagem que será composta por vários pontos denominados "pixels". Cada um dos "pixels" tem um específico coeficiente de atenuação, que associados produzirão a imagem em monitor, sendo então fotografada em filme padrão de raio-x. As imagens são apresentadas em "fatias" ou "slices" do segmento estudado, com espessuras que variam, usualmente, de acordo com o objetivo do exame, de 2 a $15 \mathrm{~mm}$. O exame axial é realizado com cortes tomográficos paralelos de uma linha formada pela borda orbitária e o meato acústico, a chamada linha órbito-meatal, sendo que em situações específicas também pode ser utilizada a técnica de cortes coronais ou de reconstrução das imagens adquiridas a partir dos cortes axiais (HAMMERSCHLAG et al., 1976).

Por ser imagem produzida graças às diferenças de densidades das estruturas estudadas, é possível a mensuração dos coeficientes de atenuação de cada uma delas. Desta forma usou-se como referencial o coeficiente de atenuação da água, que recebeu a graduação zero. A partir desse número, quanto maior a densidade da estrutura, maior é a graduação na escala, que passou a ser denominada escala de Hounsfield, escala esta que varia nas chamadas unidades Hounsfield $(\mathrm{uH})$ de -1000 até +2000uH. (HAMMERSCHLAG et al., 1976)

Von Goll e cols. (1978), analisando tomografias computadorizadas de 60 indivíduos consumidores crônicos de álcool, observaram atrofia cerebral moderada e grave em 75\% dos casos. Bergman (1983), em estudo de TC, identificou que o consumo de 50 a $60 \mathrm{~g}$ de álcool por dia estava associado a um alargamento de ventrículo em homens. Lishman (1987) encontrou em dois terços dos alcoólicos, atrofia cortical e/ou dilatação ventricular. No entanto, alguns indivíduos, apesar do tempo de dependência ou da quantidade ingerida, não apresentaram tais manifestações. O mesmo autor lembra que a idade também pode ser fator determinante de atrofia cortical, sendo difícil isolar os efeitos 
da idade dos efeitos da duração do alcoolismo, considerando que, com a idade avançada, o cérebro torna-se mais vulnerável à exposição ao álcool. Nas mulheres, encontrou exames que mostraram dilatação ventricular e atrofia cortical mais expressiva do que a identificada nos homens.

Teichman et al., em 1987, avaliando possíveis fatores envolvidos na ocorrência de atrofia cerebral em alcoolistas, analisaram o problema sob duas variáveis: a quantidade e frequência de consumo e a duração da doença.

Os resultados evidenciaram que o grau de dano cerebral está relacionado com a intensidade de exposição e claramente indicam que os testes psicológicos são métodos adequados para pelo menos realizar-se triagem objetivando o diagnóstico da atrofia cerebral.

No mesmo ano, Bergman \& Hindmarsh (1987) relataram que a dilatação dos ventrículos e dos sulcos corticais está relacionada com o tempo de consumo do álcool. No entanto, lembraram que o alargamento dos sulcos foi encontrado em todas as idades, mas em contraste, a dilatação ventricular ocorreu predominantemente nos indivíduos mais idosos. Os autores discutiram ainda a correlação positiva entre carências nutricionais e a dilatação ventricular; associação não identificada em relação ao alargamento dos sulcos corticais.

Dano \& Guyader em 1988, estudando pacientes alcoolistas com o objetivo de investigar as características da intoxicação por álcool, as complicações hepáticas e neurológicas, bem como o grau de atrofia cerebral por tomografia computadorizada, concluíram que a atrofia cerebral está relacionada com a idade e duração de intoxicação. Os autores lembraram que a atrofia ocorre predominantemente a partir de 40 anos de idade e aumenta progressivamente em paralelo com a polineuropatia, com os distúrbios cerebelares e a demência. Finalizaram os autores discutindo que tais alterações evoluem independentes dos eventuais distúrbios hepáticos.

Achados semelhantes encontraram Pfefferbaum et al. que, também no ano de 1988, analisando os efeitos morfológicos do álcool no cérebro, constataram com a tomografia computadorizada que o alargamento dos sulcos corticais estaria presente em todas as idades e que a dilatação ventricular seria verificada somente nos pacientes mais velhos.

Em 1989, Mutzell \& Tibblin, com a TC de crânio, estabeleceram que o consumo de álcool influencia nas lesões corticais e subcorticais. Discutiram a idéia de que existe no lobo frontal dos alcoolistas atrofia por eles considerada como típica e que progressivamente os doentes apresentariam lesões atróficas que iniciariam pela região frontal.

Jacobson \& Lishman (1990) encontraram nos exames de tomografia computadorizada dos cérebros de alcoolistas, a dilatação dos ventrículos laterais e do 
terceiro ventrículo, o alargamento da fissura inter-hemisférica e da fissura sylviana. Apontaram ainda os autores que a atrofia do lobo frontal é bastante acentuada e que a causa para tais lesões seria pela neurotoxicidade direta do álcool.

Harper \& Kril (1991), em estudo neuropatológico, consideraram que a atrofia cortical está relacionada a perdas dendrídicas da substância branca, mais do que da substância cinzenta. No entanto, os autores descreveram ainda importantes lesões na região frontal.

As alterações tomográficas podem ter origem no fato de que no alcoolismo, existiria uma redução dos receptores gaba-benzodiazepínicos, sendo que o cérebro das mulheres seria mais suscetível a esses efeitos. (REYNOLDS \& PRASAD, 1991)

\subsubsection{Imagem por ressonância magnética}

Na década de 80, o já conhecido princípio da ressonância magnética, que motivou dois prêmios Nobel nas décadas de 30 e 40, foi utilizado na aquisição de imagem em tecido animal. A criação de um campo magnético movimentando prótons de hidrogênio das estruturas orgânicas pôde ser transformada em imagem, pelo princípio de que cada matéria possui quantidade específica de hidrogênio que entraria em movimentação. Essa movimentação particular pode ser traduzida em imagem. (GALLUCCI et al., 1989)

Mander et al. (1989), ao analisarem as imagens cerebrais de indivíduos alcoolistas, concluíram que tal exame pode ser considerado como marcador dos danos estruturais provocados pelo alcoolismo.

Gallucci et al. (1989) avaliaram que o alcoolismo crônico pode causar lesões no sistema nervoso central, tais como, microvasculites e degenerações gliais, neurais e de mielina. Segundo os autores, deve-se esperar, também, a presença precoce de doença na substância branca cerebral, em pacientes que ainda não apresentam complicações neurológicas do alcoolismo. Por isso, concluíram os autores que a RM é de valor no diagnóstico precoce das lesões neurológicas do alcoolismo.

No entanto, Schroth et al. (1988), no ano anterior, já afirmavam que essas lesões precoces são reversíveis, visto à RM na abstinência.

A RM pode, portanto, identificar lesões menores, como isquemias e áreas de desmielinização que, usualmente, a TC não consegue visualizar. (NICHOLS \& MARTINS, 1996)

\subsubsection{Tomografia computadorizada por emissão de prótons (Pet-Scan)}

Outra modalidade de tomografia é o pet-scan. Trata-se de método diagnóstico ainda experimental na maioria dos países. Tem seu fundamento diagnóstico na mensuração do metabolismo encefálico da glicose. 
WIK e cols., em 1988, observaram a diminuição do metabolismo da glicose de até $30 \%$ em áreas corticais e subcorticais cerebrais. Afirmam ainda os autores que tais alterações não apresentaram correlação com a atrofia cortical observada pela tomografia computadorizada.

Com o mesmo exame, Lingford-Hughes e cols., em 2000, discutiram a hipótese de que no alcoolismo existiria uma redução dos receptores gaba-benzodiazepínicos e que o cérebro das mulheres seria mais suscetível a esses efeitos. Consideraram a possibilidade de existirem outras diferenças neurofarmacológicas com as consequentes implicações na farmacoterapia.

\subsection{Marcadores biológicos}

Além dos exames de imagem e eletrofisiológicos, não se pode olvidar os chamados marcadores biológicos para o alcoolismo, que são exames hematológicos utilizados não somente nos casos de intoxicação aguda, mas também nos consumidores crônicos.

O alcoolismo pode ser detectado por exames hematológicos nos casos de intoxicação aguda como também nos consumidores crônicos.

Os marcadores mais estudados são a gama-glutamil transferase; a aminotransferase aspartato e o volume corpuscular médio.

Para Allemann (2000), exceção feita ao teste CDT (Carbohydrate-deficient transferrin), cujo uso está por difundir-se, os exames clássicos recomendados e de tecnologia de execução de baixo custo e acessíveis são:

- gama-gt (gama glutamil transferase);

- VCM (volume corpuscular médio);

- TGO (transaminase glutâmico oxalacética) e

- TGP (transaminase glutâmico pirúvica).

Os exames laboratoriais podem identificar as complicações orgânicas relativas ao consumo excessivo do álcool. No entanto, como diagnóstico presuntivo, destaca-se a dosagem da gama-glutamil-transpeptase (gama GT) que pode constituir-se como a única enzima alterada no consumidor de etanol (ZEIN \& DISCOMBE, 1970). Unger \& Johnston constataram, em 1974, que o volume corpuscular médio (VCM) pode estar aumentado nos alcoólicos. Monteiro \& Masur (1986) observaram que a dosagem de gama GT e do VCM pode ter valor na monitorização do tratamento do alcoolismo e que o Gama GT é alterado mais precocemente em relação ao VCM.

No entanto, para RYB e cols (1999), ao estudarem a validade dos marcadores sanguíneos para o alcoolismo em relação à dosagem sérica do álcool, em 
pacientes admitidos em centro de trauma, concluíram que tais marcadores não acrescentam informações adicionais ao que podem trazer a entrevista psicológica e a alcoolemia.

5.6. Os testes psicológicos e suas aplicações

Os testes psicológicos são instrumentos de medida que têm por objetivo analisar a estrutura e o dinamismo psíquico.

Estão divididos em:

1. Testes de medida da inteligência;

2. Testes para o estudo da personalidade-estruturais/ temáticos;

3. Testes psicomotores.

Os testes psicológicos são importantes meios no diagnóstico e monitoração das doenças mentais, porque possibilitam o entendimento dos processos de pensamento, do funcionamento intelectual, o grau de controle frente à realidade interna e externa e as defesas utilizadas (ANDRADE \& HIRATA, 1985). Visam identificar manifestações psicopatológicas compatíveis com lesões neuronais provocadas pela bebida. (WILKINSON \& CARLEN, 1980)

Chelune (1981) e Acker (1982) observaram que os testes neuropsicológicos que apresentam maior adequação para o diagnóstico de distúrbios mentais em alcoolismo, são os que abordam a capacidade de abstração para solucionar problemas, aprendizado e memória.

Eckardt \& Martin (1986), na avaliação da função mental no alcoolismo, destacaram a importância da pesquisa da capacidade de raciocínio, planejamento, julgamento, formação de conceitos e resolução de problemas.

Parson (1987) relata que apenas uma minoria de alcoólicos não apresenta algum tipo de teste cognitivo alterado, ou seja, é frequente o achado de alterações patológicas, quando são aplicados diferentes testes psicológicos.

Com o objetivo de identificar com praticidade o consumo de álcool em populações específicas, Ewing (1984) publica artigo, fruto de estudos de vários anos, que objetivaram a "identificação do alcoolismo escondido". Esses estudos conduziram a elaboração do questionário CAGE, que, pela sua simplicidade, contendo quatro perguntas básicas, permite o diagnóstico presuntivo do alcoolismo. Masur e cols., na Universidade Federal de São Paulo - Escola Paulista de Medicina, em 1983 e 1985, utilizaram o questionário adaptando-o ao nosso meio, bem como sua aplicação em pacientes psiquiátricos e em clínica médica. Em 1985, na Faculdade de Medicina de Botucatu, KerrCorrea e cols. encontraram positividade para o teste em $9,1 \%$ dos pacientes internados naquela instituição. 
Rorschach (1911) criou a prova que, hoje, leva seu nome e que consiste na interpretação de borrões de tinta impressos em cartões, acidentalmente produzidos. O autor aplicou este teste em indivíduos normais e em outros que tinham algum diagnóstico de afecção de ordem mental, tendo observado respostas semelhantes em cada grupo específico. (EXNER, 1999) O teste de Rorschach, parte do grupo de provas atualmente conhecidas como técnicas projetivas e consiste em se colocar o sujeito numa situação mais ou menos ambígua, concedendo-lhe ampla liberdade de ação. Nos testes não há respostas certas ou erradas; cada um reage de maneira estritamente pessoal, resolvendo o estímulo de acordo com as tendências, atitudes e traços próprios e individuais. O método de Rorschach é o teste mais estudado, cuja aplicação oferece maiores garantias de êxito. Em sua aplicação se fornece ao sujeito uma série de borrões de tinta, pobremente estruturados que, entre si, pouco ou nada significam. (EXNER, 1999)

Em 1975, Fortes \& Gatto, aplicando testes de Rorschach em alcoólicos, observaram estereotipias, perseverança e caráter oral das respostas.

O teste das Pirâmides Coloridas de Pfister permite a abordagem da significação psicológica atribuída às cores, constituindo-se, com isso, meio para psicodiagnóstico. (JUSTOS, 1976)

Uma cor pode apresentar-se como um desencadeante de respostas de excitação-relaxamento, aproximação-afastamento, ou seja, uma forma de produzir um efeito sobre o organismo por inteiro e, neste sentido, a cor pode ser apreciada como estímulo. No entanto, a cor pode reapresentar-se como uma projeção de estados interiores, quando diferentes matizes são escolhidos para compor, por exemplo, uma pintura e, sob este aspecto, poderia ser apreciada como resposta. (MARQUES, 1988)

O teste do desenho da figura humana visa ao estudo da imagem corporal que é, segundo Schilder (1981), a representação mental do próprio corpo, é a imagem que todos têm de si mesmos. Considera, ainda, que a imagem corporal é inconsciente tendo relação direta com a fisiologia nervosa. Afirma também que na ocorrência de doença orgânica, surgem em termos emocionais, mudanças no conceito de si e percepção de sua identidade, que são fáceis de reconhecer no desenho da figura humana.

$O$ teste do desenho da figura humana é avaliado, considerando-se as características, sua inserção no papel, detalhes e desenvolvimento do desenho.

A interpretação psicológica do tamanho do desenho visa à identificação da relação do indivíduo com o seu meio; assim, quanto maior a figura, maior a valorização de si mesmo. Em relação à perspectiva, interpreta-se a intensidade de auto-exposição do indivíduo, isto é, o perfil de sua apresentação ao mundo. A transparência do desenho pode denotar indícios de conflitos ou negação da realidade. $\mathrm{Na}$ análise das articulações, sua presença denota deficiência no sentimento de integridade corporal. A presença da linha de solo, quando aparece, indica preocupação com a própria localização no mundo. Os 
conflitos que surgem nos desenhos podem denotar insegurança, agressividade, desejo de perfeccionismo, ansiedade, sendo que as omissões podem localizar, inclusive, áreas anatômicas de conflito. Finalmente, a figura de homem ou mulher, desenhada em primeiro lugar, pode ser considerada como o entendimento emocional, sexual e pessoal do indivíduo. (SCHILDER, 1981)

Apesar da grande acuidade dos testes psicológicos na detecção dos efeitos mentais deletérios do consumo crônico de álcool, deve-se analisá-los sob o prisma da ocasião do exame e, em sua aplicação, afastar a possibilidade do indivíduo ter feito uso recente de bebida alcoólica. O examinador deve certificar-se de que não existe efeito residual do consumo, pois assim, a análise da estrutura mental torna-se factível. (CAREY \& MAISTO, 1987)

Acheson e cols. (1998) afirmam que mesmo doses pequenas, que levariam à alcoolemia de até $0,07 \mathrm{mg} / \mathrm{dl}$, já alterariam a aquisição da memória, o que motivou a abstinência absoluta de álcool, nos dias de aplicação dos testes do presente trabalho.

Em trabalhos experimentais, Nixon e cols. (1987) e Baird e cols. (1987), afirmaram que as alterações cognitivas mais observadas foram: distúrbios da noção de localização espacial-visual; a percepção motora e memória para informações visuais e verbais.

\section{Critérios para a investigação diagnóstica}

Apesar da observação quase uniforme dos danos mentais sofridos pelos pacientes detectados pelos testes psicológicos, na população geral devem-se esperar distintos graus de intensidades de dano mental provocado pelo álcool. (HURST \& BAGLEY, 1972; THOMSON \& RON, 1982). Tal suscetibilidade individual pode também ter determinante relacionada à plasticidade neuronal. Bäckman e cols. (1998) expuseram ratos de até dez dias de nascimento a doses elevadas de etanol na dieta. Com isso, observaram histologicamente perdas de células de Purkinge, apesar de não terem sido observadas tais perdas do ponto de vista eletrofisiológico, o que denota que as poucas células de Purkinge que restaram, mantiveram a função.

Walker e cols., em 1980, experimentalmente identificaram perda neuronal na região do hipocampo de ratos submetidos à intoxicação crônica por álcool. Harper e cols., em 1985, medindo o volume da substância cinzenta cortical, da substância branca e dos gânglios da base de alcoólicos, mostrou que a perda do tecido cerebral em alcoólicos é maior na substância branca.

De La Monte, em 1988, encontrou em necropsias, atrofia desproporcional da substância branca cerebral em alcoólicos, sugerindo que o principal efeito da intoxicação crônica pelo etanol no sistema nervoso é a degeneração axonal. 
Para alguns autores como Charness (1993), as alterações neuropatológicas são mais comumente encontradas em indivíduos alcoólicos mais graves, como na síndrome de Wernicke-Korsakoff e não são encontradas usualmente em indivíduos de consumo leve a moderado de álcool. Begleiter e cols. (1982) discutiram a possibilidade de tratar-se de determinante genético que determinam a maior susceptibilidade para danos neuronais. Os autores estudaram as disfunções cerebrais de alcoólicos com ou sem história familiar de alcoolismo e observaram, através da tomografia computadorizada e de respostas auditivas, existir importantes diferenças estatísticas dos danos causados pelo álcool nos dois grupos estudados.

Pode-se estabelecer critério adjuvante para o diagnóstico de dano cerebral compatível com a "doença mental", destacada no artigo 26 do Código Penal brasileiro. A avaliação pelos potenciais de média latência chama a atenção para a grande acuidade do exame, para a detecção de alterações eletrofisiológicas cerebrais. A confirmação dos achados dos testes psicológicos, quando definimos uma população alterada em sua estrutura mental pelo alcoolismo, nos direciona no entendimento de que os potenciais evocados e, especificamente, os potenciais de média latência podem tornar-se exame de importância no diagnóstico da referida "doença mental" do Código Penal, por tratar-se de exame objetivo, que, para ser analisado, não depende de fatores relacionados à subjetividade do examinador. Trata-se de exame mensurável, de valor de prognóstico e de monitorizarão dos danos existentes. Seus achados podem ser reproduzidos por vários examinadores, no mesmo período e condições de análise, tratando-se de exame de custo relativamente baixo e não invasivo.

A associação dos testes psicológicos, exames por imagem, como a tomografia computadorizada e a ressonância magnética e, finalmente, as respostas auditivas de média latência fornecem ao perito médico, subsídios amplos para a determinação em juízo, de inimputabilidade penal dos alcoólicos crônicos.

São Paulo, março de 2010.

Referências

ABEL, E. L.; KRUGER, M. L.; FRIEDL, J. How do physicians define "light", "moderate", and "heavy" drinking? Alcoholism: Clinical and Experimental Research, Baltimore, MD, v. 5, n. 22, p. 979-984, 1998.

ACKESON, S.; STEIN, R. M.; SWANTZWELDER. Impairment of semantic and figural memory by acute ethanol age dependent effects. Alcoholism: Clinical and Experimental Research, Baltimore, MD, v. 22, n. 7 p. 1437-42, 1998. 
ALLEMANN, P. I. Marker fur ubermassigen alkoholkonsum (screening) / Markers for excessive alcohol use (screening). Ther Umsch, v. 57, n. 4, p. 185-90, abr. 2000.

ANDRADE, A. G.; HIRATA, E. S. Avaliação psiquiátrica de alcoolistas. Revista da Associação Brasileira de Psiquiatria, v. 7, n. 24, p. 17-22, 1985.

BÄCKMAN, C.; WEST, J. R.; MAHONEY, J.C.; PALMER, M. Electrophysiological caracterization of cerebellar neurons from adult rats exposed to ethanol during development. Alcoholism: Clinical and Experimental Research, Baltimore, MD, v. 22, n. 5, p. 1137-1145, 1988.

BAIRD, T. J.; VANECEK, S. A.; BRISCOL, R. J.; VALLET, M.; CARL, K. L.; GAUVIN, D. V. Moderate, long-term, alcohol consumption potentiates normal, age-related spatial memory deficits in rats. Alcoholism: Clinical and Experimental Research, Baltimore, MD, v. 22, n. 3, p. 628-36, 1998.

BEGLEITER, H.; PORJESZ, B. Persistence of brain hyperexcitability following chronic alcohol exposure in rats. Advances in Experimental Medicine and Biology, New York, n. 85, p. 209-222, 1977.

BEGLEITER, H.; PORJESZ, B.; CHOU, C. L. Auditory brain stem potentials in chronic alcoholics. Science, n. 211, p. 1064-1066, 1981.

BEGLEITER, H.; PORJESZ, B.; KISSIN, B. Brain dysfunction in alcoholism with and without a family history of alcoholism. Alcoholism: Clinical and Experimental Research, Baltimore, MD, v. 6, n. 136, 1982.

BERGMAN, H.; AXELSSON, G.; IDESTROM, C. M.; BORG, S.; HINDMARSH, T.; MAKOWER, J.; MUTZELL, S. Alcohol consumption, neurpsychological status and computer-tomographic findings in a random sample of men and women from the general population. Pharmacology Biochemisty and Behavior, New York, v. 18, n. 1, p: 501-505, 1983.

BERGMAN, H., HINDMARSH, T. Alcohol and brain damage: neuropsychological and computertomographic findings. Lakartidningen, v. 84, n. 46, p. 3828-33, nov. 1987.

BIERLEY, R. A.; CANNON, D. S.; WEHL, C. K.; DUSTMAN, R. E. Effects of alcohol on visually evoked responses in rats during addiction and withdrawal. Pharmacology Biochemisty and Behavior, New York, n. 12, p. 909-915, 1980.

BRAZIER, M. A. B. The electrical activity of the nervous system. Science, v. 146, p. 1.423-1.428, 1964.

CHARNESS, M. E. Brain lesions in alcoholics. Alcoholism: Clinical and Experimental Research, Baltimore, MD, v. 17, p. 2-11, 1993.

CHELUNE, G. J.; PARKER, J. B. Neuropsychological deficits associated with chronic alcohol abuse. Clinical Psychology Review, Arlington, n. 1, p. 181-195, 1981.

CHU, N. S.; SQUIRES, K. C.; STARR, A. Auditory brain stem potentials in chronic alcohol intoxication and alcohol withdrawal. Archives Neurology, Chicago, n. 35, p. 596, 1978. 
CHURCH, M. W.; WILLIAMS, H. L. Dose and time-dependent effects of ethanol brains stem auditory evoked responses in young adult males. Electroencephalography and Clinical Neurophysiology, Limerick, n. 54, p. 161-174, 1982.

CLARREN, S. K.; SMITH, D. W. The fetal alcohol syndrome: A review of the world literature. The New England Journal of Medicine, Walthan, n. 298, p. 1063-1067, 1978.

CORBETT, C. E. Hipnóticos. In: CORBETT, C. E. Farmacodinâmica. 6. ed. Rio de Janeiro: Guanabara Koogan, 1982.

DANO, P.; LE GUYADER, J. Atrophie cerebrale et alcoolisme chronique / Cerebral atrophy and chronic alcoholism. Revue Neurologique, Paris, v. 144, n. 3, p. 202-208, 1988.

DE LA MONTE, S. M. Disproportionate atrophy of cerebral with matchs chronic alcoholics. Archives Neurology, Chicago, v. 45, p. 990-992, 1988.

DIAMOND, I.; GORDON, A. S. Cellular and molecular neuroscience of alcoholism. Physiological Review, n. 77, p. 1-20, 1997.

DOM ZAL, T., KOTOWICZ, J., WALECKI, J. et al. Clinical, CT and eletrophysiologic studies of alcoholics with special reference to the cerebellum. Neurol. Neurochir. Pol., v. 23, n. 1, p. 17-20, jan. 1989.

ECKARDT, M. J.; MARTIN, P. R. Clinical assessments of cognition on alcoholism. Alcoholism: Clinical and Experimental Research, Baltimore, n. 10, p. 123-127, 1986.

ELKIS, H. Definições e critérios para o diagnóstico de alcoolismo. Jornal Brasileiro de Psiquiatria, Rio de Janeiro, v. 34, p. 319-322, 1985.

EXNER JN., J. Manual de classificação de Rorschach. São Paulo: Casa do Psicólogo, 1999. 322 p.

FORTES, J. R. A.; GATTO, B. C. F. Alcoolismo. São Paulo: Savier, 1975. 322 p.

GALLUCCI, M.; AMICARELLI, I.; ROSSI, A. et al. MR imaging of white matter lesions in uncomplicated chronic alcoholism. Journal of Computer Assisted Tomography, New York, v. 13, n. 3, p. 395-398, maio/jun. 1989.

GANANÇA, M. M. Vertigem tem cura? São Paulo: Lemos Editorial, 1998. 301 p.

GLASS, A. Mental arithmetic and blocking of the occipital alpha rhythm. Electroencephalography and clinical neurophysiology, Limerick, v. 16, p. 595-603, 1964.

GUEBALY, N.; GUEBALY, A. Alcohol in ancient Egypt: the recorded evidence. Addict., v. 16,p. 1027-1221, 1981.

HALL III, J. W. Handbook of auditory evoked responses. Allyn and Bacon: ed. Needham Heights, 1990.

HAMMERSCHLAG, S. B.; WOLPERT, S. M.; CARTER, B. L. Computed coronal tomography. Radiology, v. 120, p. 219-220, 1976. 
HARPER, C. KRILL, J.; DALY, J. Does a moderate alcohol intake damage brain? Journal of Neurology Neurosurgery \& Psychiatry, v. 51, p. 909-913, 1988.

HARPER, C., KRIL, J. If you drink your brain will shrink; neuropathological considerations. Alcohol and Alcoholism: supplement, Oxford, v. 1, p. 375-80, 1991.

HOUNSFIELD, G. N. Computerized transverse axial scanning (tomography); 1.description of system. The British Journal of Radiology Archives, London, v. 46, p. 1.016-1.022, 1973.

HURST, P. M. \& BAGLEY, S. K. Acute adaptation to the effects of alcohol. Quarterly Journal of Studies on Alcohol, New Haven, n. 33, p. 358-378, 1972.

HYVARINEN, J.; LAAKSO, M.; ROINE, R.; LEINOMEN. L.; SIPPEL, H. Effects of ethanol on neural activity in the parietal association cortex of alert monkeys. Brain, Oxford, n. 101, p. 701-715, 1978.

ISHIBASHI, M.; NAKAZAWA, Y.; YOKOYAMA, T. et al. Cerebral atrophy and slow wave sleep of abstinent chronic alcoholics. Drug Alcohol Dependence, v. 19, n. 4, p. 325-332, jul. 1987.

JÄÄSKELÄINEN, I. P.; LEHTOKOSKI, A.; ALHO, K.; KUJALA, T.; PEKKONEN, E.; SINCLAIR, J.D.; NÄÄTÄNEN, R.; SILLANAUKEE, P. Low dose of ethanol suppresses mismatch negativity of auditory event-related potentials. Alcoholism: Clinical and Experimental Research, Baltimore, n. 19, p. 607-610, 1995.

JACOBSON, R. R.; LISHMAN, W. A. Cortical and diencephalic lesions in Korsakoff's syndrome: a clinical and CT scan study. Psychological Medicine, Cambridge, v. 20, n. 1, p. 63-75, fev. 1990.

JELLINEK, E. M. Phases in drinking history of alcoholics. Quarterly Journal of Studies on Alcohol, New Haven, n. 7, p. 1-88, 1946.

JELLINEK, E. M. Drinkers and alcoholics in ancient Rome. Quarterly Journal of Studies on Alcohol, New Haven, n. 37, p. 1718-1741, 1976.

JUSTOS, H.; VANKOLCH, T. O teste das pirâmides coloridas de cores. São Paulo: Vetor, 1976.

KELLER, M. Concepções sobre o alcoolismo. Revista da Associação Brasileira de Psiquiatria, São Paulo, v. 2, p. 93-100, 1983.

KRAMER, S. N. Mesopotâmia: o berço da civilização. In: BIBLIOTECA DE HISTÓRIA UNIVERSAL LIFE. Rio de Janeiro: J. Olympio, 1983.

KRAUSS, G. L.; NIEDERMEYER, E. Electroencephalogram and seizures in chronic alcoholism. Electroencephalography and Clinical Neurophysiology, Limerick, v. 78, n. 2, p. 97-104, fev. 1991.

LEE, K. Alcoholism and cerebrovascular thrombosis in the young. Acta Neurologica Scandinavica, Copenhagen, n. 59, p. 270-274, 1979.

LEE, K.; JENSEN, E.; BECH, P. Nuerophysiological and computadorized tomographic evaluations of young alcoholics. The British Journal of Psychiatry, London, n. 141, p. 282-285, 1982. 
LINNOILA, M.; STAPLETON, J.; LISTER, R.; GUTHRIE, S.; ECKARDT, M. Effects of alcohol on accident risk. Pathologist, n. 40, p. 36-41, 1986.

LISHMAN, W. A. Brain damage in alcoholism: currents. Acta Medica Scandinavica: supplement, Copenhagen, n. 717, p. 5-17, 1987.

LOVINGER, D. M. Excitotoxicity and alcohol-related brain damage. Alcoholism: Clinical and Experimental Research, Baltimore, n. 17, p. 19-27, 1993.

LUZINSE, J.; ZIMBERG, S.; SMOKLER, H. GURLEY, K. Alcoholism and cerebral atrophy: a study of 50 patients with CT-scan and psychologic testing. Alcoholism: Clinical and Experimental Research, Baltimore, n. 4, p. 405-411, 1980.

MANDER, A. J., YOUNG, A., CHICK, J. D., BEST, J. J. The relationship of cerebral atrophy and T1 in alcoholics: an MRI study. Drug Alcohol Dependence, v. 24, n. 1, p. 57-59, ago. 1989.

MARQUES, M. I. B. O teste das pirâmides coloridas de Max Pfister. São Paulo: EPU, 1988.

MILNER, B. Effects of different brain lesions on card sorting. Archives of Neurology, Chicago, v. 9, p. 90-100, 1963.

MONTEIRO, M. G.; MASUR, J. Monitoring alcoholism treatment: the appropriateness of choice between GGT and MCV evaluation after a short time of abstinence. Alcohol, n. 3, p. 223-226, 1986.

MORSE, R. M.; FLAVIN, D. K. The definition of alcoholism. JAMA: Journal of American Medical Association, Chicago, v. 268, n. 8, p. 1012-1014, 1992.

MUTZELL, S., TIBBLIN, G. High alcohol consumption, liver toxic drugs and brain damage - a population study. Upsala Journal of Medical Sciences, v. 94, n. 3, p. 305-315, 1989.

NATIONAL COUNCIL ON ALCOHOLISM / AMERICAN MEDICAL SOCIETY ON ALCOHOLISM COMMITTEE ON DEFINITIONS. Definition of alcoholism. 1992. Annals of Internal Medicine, v. 85, p. 764, 1976.

NICHOLS, J. M.; MARTIN F. P300 and heavy social drinkers: the effect of lorazepan. Alcohol, v. 10, p. 269-274, 1993.

NICHOLS, J. M.; MARTIN, F. The effect of heavy social drinking on recall and event-related potentials. Journal of Studies on Alcohol, v. 57, p. 125-135, 1996.

NIXON, S.J.; KUJAWSKI, A.; PARSONS, O.A.; YOHMAN, J. R. Semantic (verbal) and figural memory impairment in alcoholics. Journal of Clinical and Experimental Neuropsychology, London, v. 9, p. 311-322, 1987.

OGURA, C., MIYAZATO, Y. Cognitive dysfunctions of alcohol dependence using event related potentials. Arukoru Kenkyuto Yakubutsu Ison, v. 26, n. 5, p. 331-340, out. 1991.

PACHECO E SILVA, A. C. Psiquiatria Clínica e Forense. 2. ed. São Paulo: Renascença, 1951. 
PEEKE, S. C.; PRAEL, A. R.; HERNING, R. I.; ROGERS, W.; BENOWITZ, N. L.; JONES, R. T. Effect of disulfiram on cognition, subjective response, and cortical-event-related potentials in nonalcoholic subjects. Alcoholism, v. 3, p. 223-229, 1979.

PEKKONEN, E.; AHVENINEN, J.; JÄÄSKELÄINEN, L. P.; SEPPÄ, K.; NÄÄTÄNEN, R.; SILLANAUKEE, P. Selective acceleration of auditory processing in chronic alcoholics during abstinence. Alcoholism: Clinical and Experimental Research, Baltimore, v. 22, n. 3, p. 605-609, 1998.

PFEFFERBAUM, A., HORVATH, T. B., ROTH, W. T. et al. Event-related potential changes in chronic alcoholics. Electroencephalography and Clinical Neurophysiology, Limerick, v. 47, p. 637647, 1979.

PFEFFERBAUM, A., ROSENBLOOM, M., CRUSAN, K. et al. Brain CT changes in alcoholics: effects of age and alcohol consumption. Alcoholism: Clinical and Experimental Research, Baltimore, v. 12, n. 1, p. 81-87, fev. 1988.

PORJESZ, B.; BEGLEITER, H. Human evoked brain potentials an alcohol. Alcoholism: Clinical and Experimental Research, Baltimore, v. 2, n. 5, p. 304-316, 1989.

REAlMUtO, G.; BEGlEITER, H.; ODENCRANZ, J.; PORJEZ, B. Event-related potentials evidence of dysfunction in automatic processing in abstinent alcoholics. Biological Psychiatry, v. 33, p. 594-601, 1993.

REYNOLDS, J. N.; PRASAD, A. Ethanol enhances GABAA receptor activated chloride currents in chick cerebral cortical neurons. Brain Research, v. 564, p. 138-142, 1991.

ROMANO, J.; ENGEL, G. L. Delirium: I. Electroencephalographic data. Archives of Neurology and Psychiatry, v. 51, p. 356-377, 1944.

RON, M.A. Brain damage in chronic alcoholism. A neuropathological, neuroradiological and psychological review. Psychological Medicine, Cambridge, v. 7, p. 103-112, 1977.

RYB, G. E.; SODERSTROM, C. A.; KUFERA, J. A. et al. Use of blood alcohol concentration and laboratory tests to detect current alcohol dependence in trauma center patients. Journal of Trauma, v. 47 , n. 5, p. 874-880, nov. 1999.

SALAMY, A. The effects of alcohol on the variability of the human evoked potential. Neuropharmacology, v. 12, p. 1.103-1.107, 1973.

SALAMY, A.; WILLIAMS, H. L. The effects of alcohol on sensory evoked and spontaneous cerebral potentials in man. Electroencephalography and Clinical Neurophysiology, Limerick, v. 35, p. 3-11, 1973.

SCHILDER, P. A imagem do corpo. 1. ed. São Paulo: Martins Fontes, 1981.

SCHROTH, G., NAEGELE, T., KLOSE, U. et al. Reversible brain shrinkage in abstinent alcoholics, measured by MRI. Neuroradiology, v. 30, n. 5, p. 385-389, 1988. 
SCHUCKIT, M. A. A clinical review of alcohol, alcoholism and the elderly patient. Journal of Clinical Psychiatry, v. 43, p. 396-399, 1982.

SILVERMAN, D. Some observations on the EEG in hepatic coma. Electroencephalography and Clinical Neurophysiology, Limerick, v. 14, p. 53-59, 1962.

SILVERMAN, D. Retrospective study of the EEG in coma. Electroencephalography and Clinical Neurophysiology, Limerick, v. 15, p. 486-503, 1963.

SQUIRES, K. C.; CHU, N. S.; STARR, A. Acute effects of alcohol on auditory brainstem potentials in humans. Science, v. 201, p. 174-176, 1978 b.

STOCKARD, J. J.; ROSSITER, V. S. Clinical and pathologic correlates of brainstem auditory response abnormalities. Neurology, 27: 316-325, 1977.

TEICHMAN, M.; RICHMAN, S.; FINE, E.W. "Dose/duration effect" relationship between alcohol consumption and cerebral atrophy: a psychological and neuroradiological evaluation. American Journal of Drug and Alcohol Abuse, v. 13, n. 3, p. 357-363, 1987.

THOMSON, A. D.; RON, M. A. Alcohol-related structural brain. British Medical Bulletin, v. 38, p. 87-94, 1982.

UNGER, K. W.; JOHNSTON, D. J. Red blood cellmean corpuscular volume a potential indicator of alcohol usage in a working population. American Journal of the Medical Science, v. 267, p. 281289, 1974.

VON GOLL, M.; BECKER, H.; ARTMANN, H.; LERCH, G. Results of computer tomography on chronic alcoholics. Neurology, v. 16, p. 329-331, 1978.

WALKER, D. W.; BARNES, D. E.; ZORNEYZER, S. F.; HUNTER, B. E.; KUBANIS, P. Neuronal loss in hippocampus induced by prolonged ethanol consumption in rats. Science, v. 209, p. 711-713, 1980.

WALKER, J. I. Psychiatric emergencies. Intervention and resolution. Philadelphia: Lippincott, 1983.

WEST, L. J.; MAXWELL, D. S.; NOBLE, E. P.; SOLOMOM, D. H. Alcoholism. Annals of Internal Medicine, v. 100, p. 405-416, 1984.

WIK, G.; BORG, S.; SJOGREN, I. et al. PET determination of regional cerebral glucose metabolism in alcohol-dependent men and healthy controls using 11C-glucose. Acta Psychiatrica Scandinavica, v. 78, n. 2, p. 234-241, ago. 1988.

ZEIN, M.; DISCOMBE, G. Serum gamma glutamyl transpeptidase as a diagnostic aid. Lancet, v. 2, p. 748-750, 1970.

ZILM, D. H.; HUSZAR, L.; CARLEN, P. L.; KAPLAN. H. L.; WILKINSON, D. A. EEG correlates of the alcohol-induced organic brain syndrome in man. Clinical Toxicology, v. 16, p. 345-358, 1980. 